\title{
Trichostatin A, a histone deacetylase inhibitor, suppresses proliferation and promotes apoptosis of esophageal squamous cell lines
}

\author{
JUNFEN MA $^{1 *}$, XIAOBING GUO ${ }^{1 *}$, SHIJIE ZHANG ${ }^{1}$, HONGCHUN LIU $^{1}$, \\ JING LU ${ }^{2}$, ZIMING DONG ${ }^{2}$, KANGDONG LIU ${ }^{2}$ and LIANG MING ${ }^{1}$ \\ ${ }^{1}$ Department of Clinical Laboratory, The First Affiliated Hospital of Zhengzhou University, Zhengzhou, Henan 450052;
${ }^{2}$ Department of Pathophysiology, School of Basic Medical Sciences, Zhengzhou University, Zhengzhou, Henan 450001, P.R. China
}

Received April 27, 2014; Accepted January 9, 2015

DOI: $10.3892 / \mathrm{mmr} .2015 .3268$

\begin{abstract}
Histone deacetylase (HDAC)-mediated epigenetic modification plays crucial roles in numerous biological processes, including cell cycle regulation, cell proliferation and apoptosis. HDAC inhibitors demonstrate antitumor effects in various cancers, including glioblastoma and breast cancer. HDAC inhibitors are therefore promising antitumor drugs for these tumors. The tumorigenesis and development of esophageal squamous cell carcinoma (ESCC) involve genetic and epigenetic mechanisms. However, the effects of the HDAC inhibitor on ESCC are not fully investigated. In the present study, ESCC cells were treated with trichostatin A (TSA) and its antitumor effects and related mechanisms were investigated. The results indicated that TSA suppressed the proliferation of ESCCs and caused G1 phase arrest by inducing the expression of p21 and p27. TSA also induced cell apoptosis by enhancing the expression of pro-apoptotic protein Bax and decreasing the expression of anti-apoptotic protein Bcl-2. Furthermore, TSA inhibited the expression of phosphatidylinositol-3-kinase (PI3K) and reduced the phosphorylation of Akt and extracellular signal-regulated kinase (ERK)1/2 in EC9706 and EC1 cell lines. High levels of acetylated histone $\mathrm{H} 4$ were detected in TSA-treated ESCC cell lines. Overall, these results indicate
\end{abstract}

Correspondence to: Dr Kangdong Liu, Department of Pathophysiology, School of Basic Medical Sciences, Zhengzhou University, 100 Kexue Avenue, Zhengzhou, Henan 450001, P.R. China

E-mail:kdliu@zzu.edu.cn

Dr Liang Ming, Department of Clinical Laboratory, The First Affiliated Hospital of Zhengzhou University, 1 Jianshe Road, Zhengzhou, Henan 450002, P.R. China

E-mail: mingliang@zzu.edu.cn

*Contributed equally

Key words: histone deacetylase inhibitor, trichostatin A, esophageal squamous cell carcinoma, apoptosis that TSA suppresses ESCC cell growth by inhibiting the activation of the PI3K/Akt and ERK1/2 pathways. TSA also promotes cell apoptosis through epigenetic regulation of the expression of apoptosis-related protein.

\section{Introduction}

Esophageal squamous cell carcinoma (ESCC) is a highly malignant and lethal disease due to its late diagnosis, the high incidence of post-surgical local-regional recurrence, and frequent distant metastasis. Although therapeutic methods have been improved, the 5-year survival rate is still only approximately $20 \%$ (1-2). Currently, a combination of cisplatin and 5-fluorouracil (5-FU) is frequently used to treat ESCC patients in clinical practice. However, the outcome is unsatisfactory due to its limited effects and side-effects, including nausea, vomiting and myelosuppression. In addition, certain patients are resistant to the radical chemotherapy (3). Therefore, new drugs are required to improve the clinical outcome and decrease the tolerance of ESCC to chemotherapy.

Epigenetic mechanisms including DNA methylation and histone modification play significant roles in the initiation and progression of cancer. The histone deacetylase (HDAC) is an enzyme which removes acetyl groups from histone and non-histone proteins, which will lead to chromatin remodeling. HDACs play crucial roles in numerous biological processes, including cell cycle regulation, cell proliferation and differentiation (4-5). HDACs have been observed to be overexpressed in a number of tumor types, suggesting that HDACs are potential targets for epigenetic treatment (6-7).

HDACs are a multiclass family consisting of 18 human HDACs, which are divided into four major classes: class I HDACs including HDAC 1, 2, 3 and 8; class II HDACs including HDAC 4, 5, 6, 7, 9 and 10; class III HDACs including SIRT1, 2, 3, 4 and 5; and class IV HDACs including HDAC 11 (8-9). Trichostatin A (TSA), a known class I and II HDAC inhibitor, has been demonstrated to exert multiple antitumor effects (10-12). It is reported that TSA strongly inhibits cell proliferation and induces cell cycle arrest, and subsequently induces cell apoptosis (13-15). However, the effect of TSA on ESCC cells has not been characterized. 
In this study, we report on the inhibition of the proliferation of ESCC cells by TSA through cell cycle arrest and cell apoptosis. We further investigate the mechanism involved in this process by analyzing cell cycle regulators p21 and p27 as well as apoptotic protein markers Bcl-2 and Bax. In addition, we analyze the expression of phosphatidylinositol-3-kinase (PI3K)/Akt and extracellular signal-regulated kinase (ERK)1/2, and the level of histone $\mathrm{H} 4$ acetylation before and after TSA treatment to reveal the mechanism of epigenetic modification.

\section{Materials and methods}

Cell lines and cell culture. The EC9706 cell line was a gift from the State Key Laboratory of Molecular Oncology, Chinese Academy of Medical Science (Beijing, China). The EC1 cell line was kindly provided by the University of Hong Kong (Hong Kong, China). The cell lines were propagated in monolayer culture in RPMI-1640 medium supplemented with $10 \%$ fetal bovine serum $\left(56^{\circ} \mathrm{C}, 30 \mathrm{~min}\right), 1 \times 10^{5} \mathrm{U} / 1$ penicillin and $100 \mathrm{mg} / 1$ streptomycin in a humidified atmosphere with a $5 \% \mathrm{CO}_{2}$ incubator at $37^{\circ} \mathrm{C}$. The present study was approved by the Medical Ethics Committee of Zhengzhou University (Zhengzhou, China).

Reagents and treatment. TSA was purchased from Sigma (St. Louis, MO, USA). It was dissolved in dimethyl sulfoxide (DMSO; Sigma-Aldrich) as a $5 \mu \mathrm{M}$ stock solution, and stored at $-20^{\circ} \mathrm{C}$. Control cells were treated with DMSO in parallel in each experiment. Mouse monoclonal antibodies to p21, p27, Bcl-2 and Bax, and rabbit monoclonal antibodies to PI3K, p-Akt, Akt, ERK1/2 and acetyl-histone H4 (Lys8) were purchased from Santa Cruz Biotechnology, Inc. (Santa Cruz, CA, USA); horse anti-mouse and goat anti-rabbit horseradish peroxidase-conjugated secondary antibodies were purchased from Cell Signaling Technology, Inc. (Danvers, MA, USA). The CCK-8 kit was purchased from Dojindo Laboratories (Kumamoto, Japan). The Annexin V-FITC kit was purchased from Beckman Coulter (Miami, FL, USA).

Cell viability assay. ESCC cell lines were seeded at a density of $5 \times 10^{3}$ in 96 -well microtiter plates. After culturing for $24 \mathrm{~h}$, cells were treated with TSA at various concentrations $(0.1,0.3,05,1.0,3.0$ and $5.0 \mu \mathrm{M})$ prepared from a stock solution dissolved in DMSO for 24 and 48 h, respectively. Cells treated with identical concentrations of DMSO (diluent for depsipeptide) were used as control. Four hours before measuring the absorbance, $10 \mu \mathrm{l}$ CCK-8 solution was added to each well and incubated. The absorbance at $450 \mathrm{~nm}$ wavelength was determined for each well using an enzyme-labeling instrument. All studies were performed in triplicate independently.

Cell cycle analysis. Cells were treated with various concentrations of TSA $(0.3,0.5$ and $1.0 \mu \mathrm{M})$ for $48 \mathrm{~h}$, then cells were harvested with $2.5 \mathrm{~g} / 1$ trypsin and fixed in absolute ethanol overnight at $4{ }^{\circ} \mathrm{C}$. The cells were resuspended in phosphate-buffered saline (PBS) containing 1\% RNase and then $5 \mu \mathrm{g} / \mathrm{ml}$ propidium iodide (PI) was added. Cells were incubated in the dark for $15 \mathrm{~min}$ at room temperature. A total of $3 \times 10^{4}$ cells were counted using a flow cytometer.
Apoptosis assay. Following incubation with or without TSA for $48 \mathrm{~h}$, ESCC cells were harvested with $2.5 \mathrm{~g} / 1$ trypsin and washed twice with PBS. A total of $1 \times 10^{5}$ cells were stained with fluorescein isothiocyanate (FITC)-Annexin V-PI using the Annexin V-FITC kit (Beckman Coulter) according to the manufacturer's instructions. At least $1.5 \times 10^{4}$ cells were counted by flow cytometric analysis. Each experiment was performed in triplicate. The percentage of apoptotic cells was calculated with CellQuest 3.0 software (BD Biosciences, Franklin Lakes, NJ, USA). Cells that were stained negatively with Annexin V and PI were considered viable cells. Early apoptotic cells were positive for Annexin V and negative for PI, and late apoptotic cells were positive for Annexin V and PI.

Western blot analysis. Cells were washed with cold PBS twice and lysed in lysis buffer. After $20 \mathrm{~min}$ on ice, the lysates were centrifuged at $14000 \mathrm{rpm}$ at $4^{\circ} \mathrm{C}$ for $10 \mathrm{~min}$. The supernatants were used as whole cell extracts. The protein concentration of cells was analyzed using the BCA protein assay kit (Pierce Biotechnology, Inc., Rockford, IL, USA). Cell lysate (50 $\mu \mathrm{g})$ was mixed with $5 \mathrm{X}$ sodium dodecyl sulfate (SDS) sample buffer, separated on a $10 \%$ SDS-polyacrylamide gel and transferred to polyvinylidene fluoride membranes. Membranes were blocked with $5 \%$ non-fat milk for $1 \mathrm{~h}$ and incubated with the primary antibodies at $4{ }^{\circ} \mathrm{C}$ overnight. The following primary antibodies and dilutions were used: p21 (1:500), p27 (1:500), Bcl-2 (1:1000), Bax (1:1000), PI3K (1:200), p-Akt (1:500), Akt (1:500), p-ERK1/2 (1:1000), ERK1/2 (1:1000) and acetylated histone 4 (AH4; 1:1000). The membranes were then washed with PBS-Tween $(0.1 \%)$ three times, each for $5 \mathrm{~min}$. Next, membranes were incubated with a 1:5000 dilution of horseradish peroxidase conjugated with either goat antimouse or goat antirabbit for $2 \mathrm{~h}$ at room temperature. Target proteins were detected with an enhanced chemiluminescence detection kit (ECL, Pierce). Protein levels were quantified with Quantity One ${ }^{\circledR}$ software (Bio-Rad, Hercules, CA, USA).

Statistical analysis. Data were expressed as the means \pm standard deviation, and statistical analysis was performed by analysis of variance using SPSS 13.0 software (SPSS, Inc., Chicago, IL, USA). One-way analysis of variance was used to compare differences among groups. $\mathrm{P}<0.05$ was considered to indicate a statistically significant difference.

\section{Results}

TSA induces morphological change and inhibits cell viability of ESCC cell lines. Morphological changes of ESCC cells were examined via microscopy. As shown in Fig. 1B, ESCC cells treated with control (DMSO) grew in clusters and were confluent. However, cells treated with $1.0 \mu \mathrm{M}$ TSA lost the normal cell morphology and had flattened and spindle shapes. The proliferative ability of the ESCC cells treated with various concentrations of TSA $(0.1-5.0 \mu \mathrm{M})$ was assessed by 3-(4,5-dimethylthiazol-2-yl)-2,5-diphenyltetrazolium bromide (MTT) assay. The proliferation of EC9706 was not significantly inhibited by TSA at doses of $0.1,0.3$ or $0.5 \mu \mathrm{M}$ after 24 and $48 \mathrm{~h}$. However, the proliferation of EC9706 was notably inhibited at doses over $1.0 \mu \mathrm{M}$ (Fig. 1A). The cell proliferation analysis revealed that EC1 cells were more sensitive to 

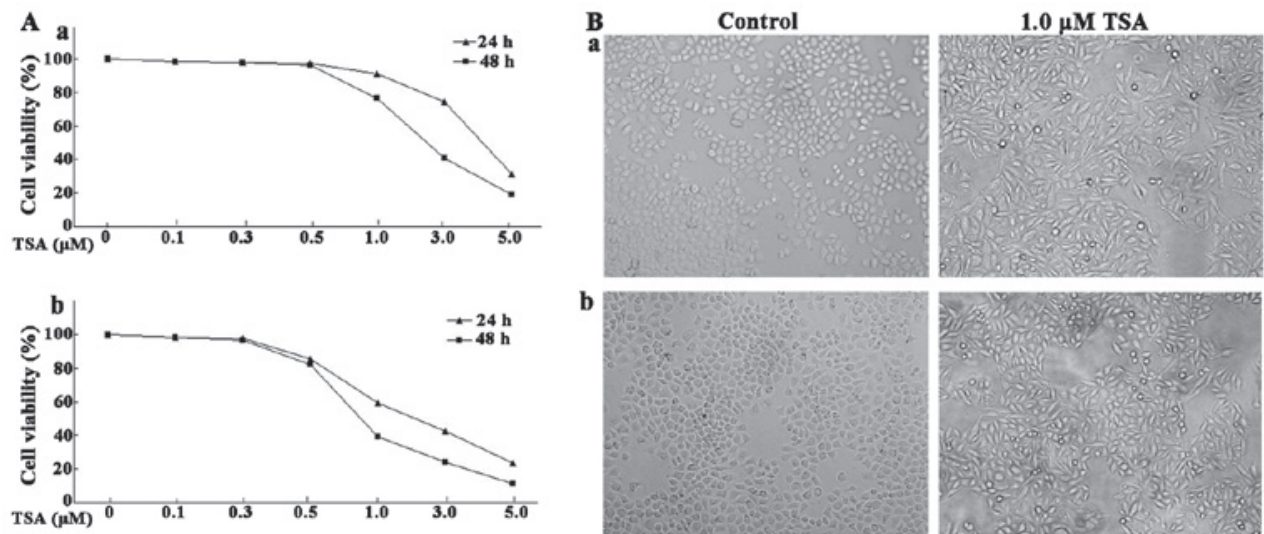

Figure 1. Effects of trichostatin A (TSA) on morphological change and cell viability in esophageal squamous cell carcinoma (ESCC) cell lines. (A) Growth inhibition of ESCC cell lines by TSA. (a) EC9706 and (b) EC1 cells were treated with TSA at indicated concentrations $(0.1-5.0 \mu \mathrm{M})$ for 24 and 48 h. Cell viability of ESCC cells was determined by CCK- 8 assay. Results shown are the means \pm SD of three independent experiments performed in triplicate. (B) TSA induces morphological changes in ESCC cells. (a) EC9706 and (b) EC1 cells were treated with control or $1.0 \mu \mathrm{M}$ TSA for $48 \mathrm{~h}$ and examined with light microscopy (x200 magnification) for changes in cell morphology.
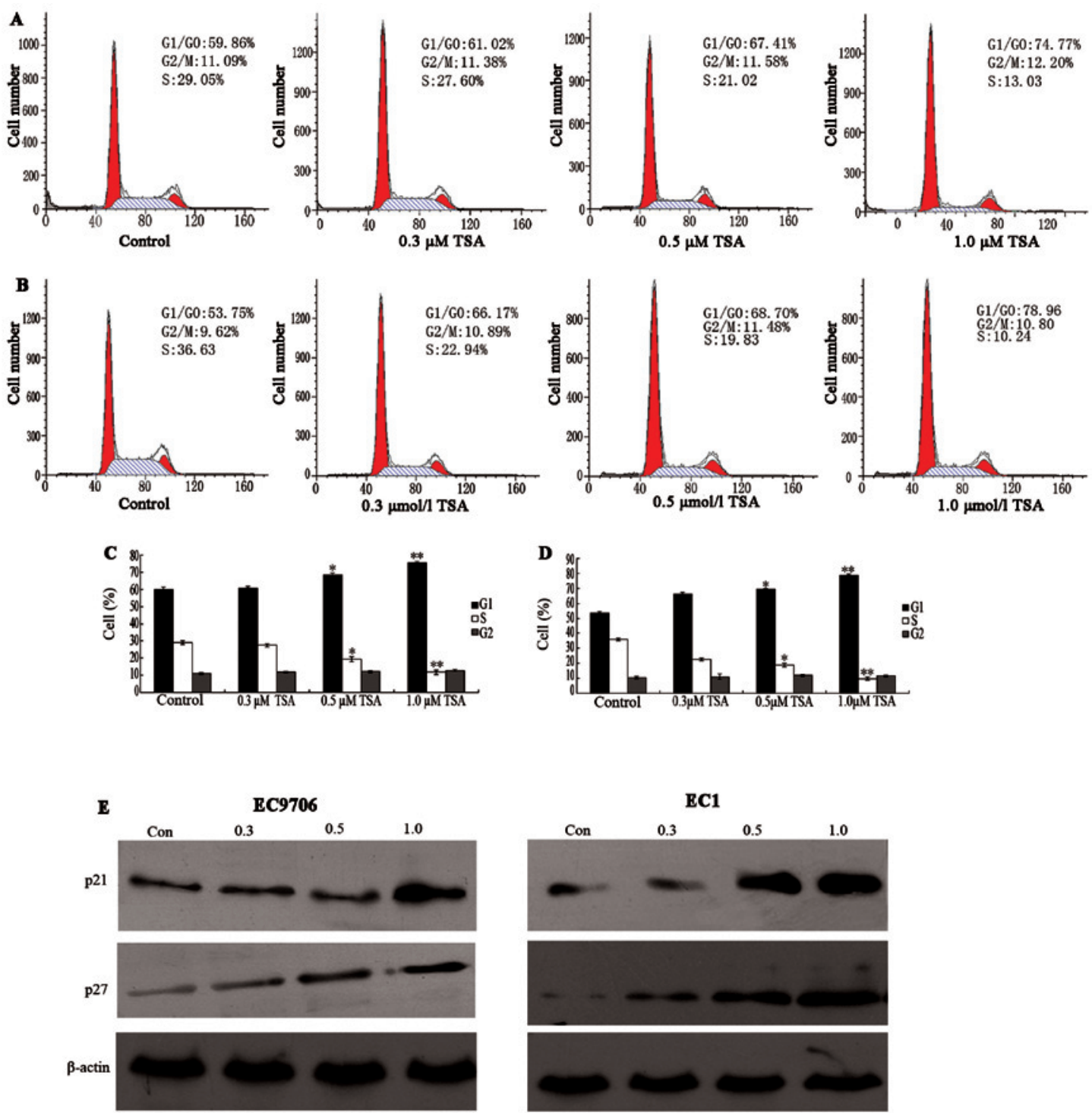

Figure 2. Effects of trichostatin A (TSA) on cell cycle in esophageal squamous cell carcinoma cell lines. (A) EC9706 and (B) EC1 cells were treated with TSA $(0.3,0.5$ and $1.0 \mu \mathrm{M})$ for $48 \mathrm{~h}$. Propidium iodide staining and flow cytometric analysis of cell cycle distribution in two cell lines above. Relative changes in the percentage of (C) EC9706 and (D) EC1 cells in each phase of the cell cycle following treatment with TSA $(0.3,0.5$ and $1.0 \mu \mathrm{M})$ for $48 \mathrm{~h}$. Results shown are the means \pm SD of three independent experiments performed in triplicate. ${ }^{*} \mathrm{P}<0.05$ and ${ }^{* *} \mathrm{P}<0.01$ compared with vehicle treatment. (E) Protein expression levels of p21 and p27 detected by western blot analysis following treatment with TSA for $48 \mathrm{~h}$ in EC9706 and EC1 cell lines. All experiments were repeated three times. 

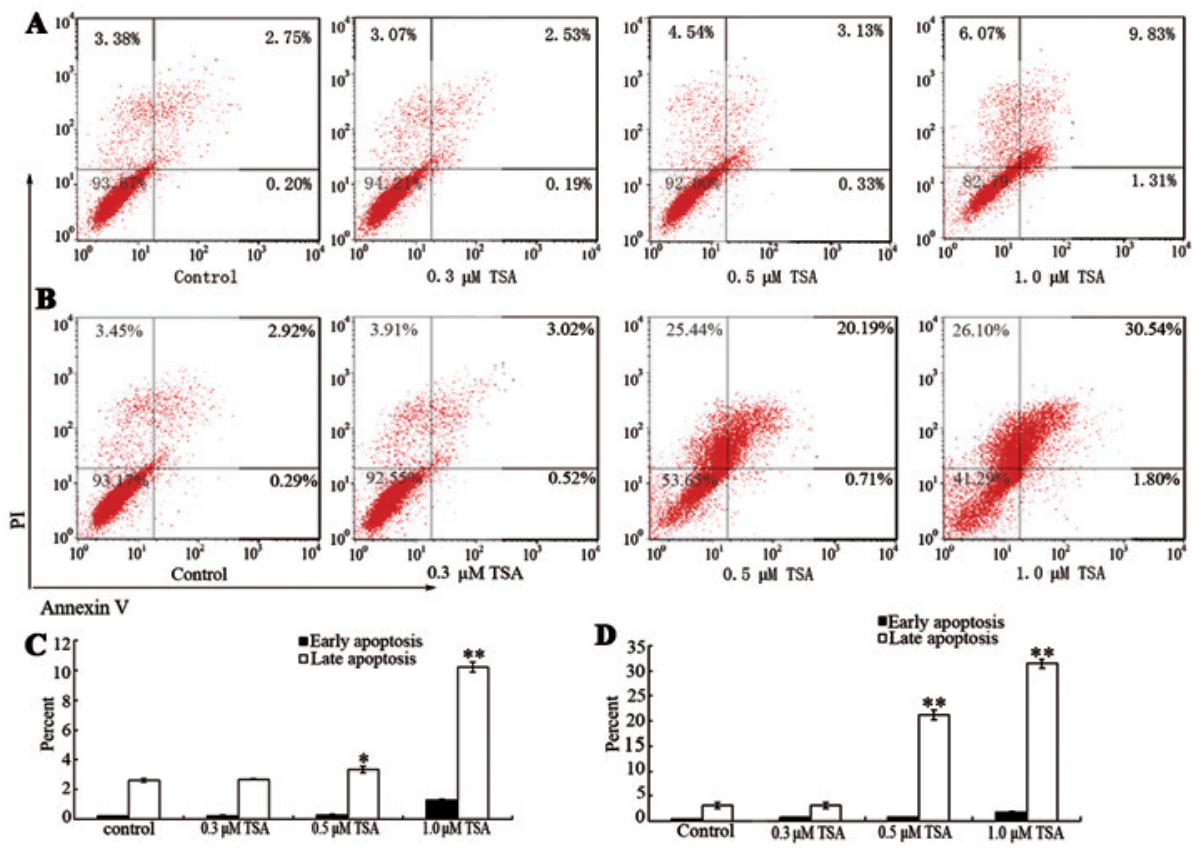

$0.5 \mu$ II TSA $1.0 \mu$ MI TSA
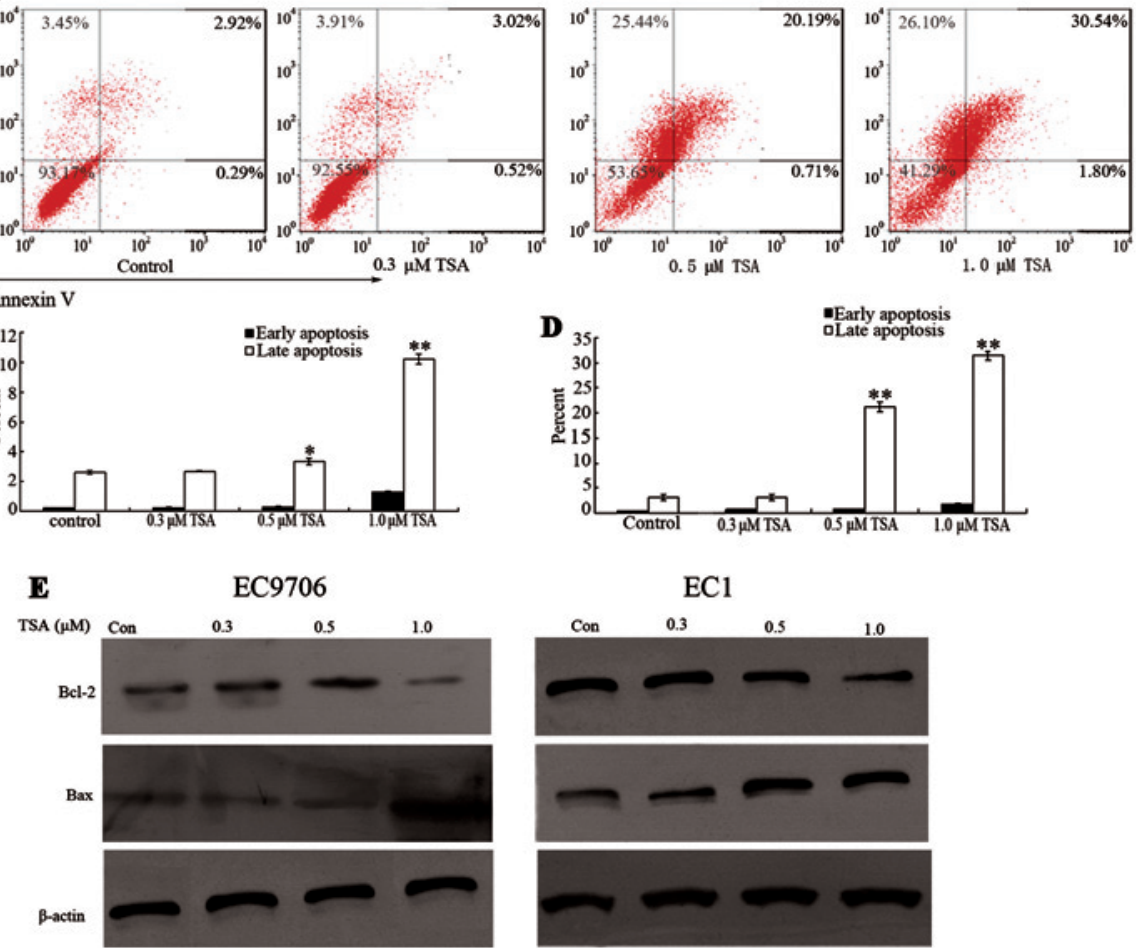

Figure 3. Effects of trichostatin A (TSA) on cell apoptosis in esophageal squamous cell carcinoma cell lines. (A) EC9706 and (B) EC1 cells were treated with TSA $(0.3,0.5$ and $1.0 \mu \mathrm{M})$ for $48 \mathrm{~h}$. (A) EC9706 and (B) EC1 cells were treated with TSA (concentrations as indicated) for $48 \mathrm{~h}$ then stained with Annexin V/PE-7AAD dual and analyzed with flow cytometric analysis. The percentage of (C) EC9706 and (D) EC1 cells that were in the early and late apoptotic stages following treatment with TSA $(0.3,0.5$ and $1.0 \mu \mathrm{M})$. Results shown are the means \pm SD of three independent experiments performed in triplicate. ${ }^{*} \mathrm{P}<0.05$ and ${ }^{* *} \mathrm{P}<0.01$ compared with vehicle treatment. (E) Protein expression levels of Bcl-2 and Bax were detected by western blot analysis following treatment with TSA for $48 \mathrm{~h}$ in EC9706 and EC1 cell lines. All experiments were repeated three times with similar results.

TSA treatment than EC9706 cells. After $24 \mathrm{~h}$ of treatment at the dose of $5.0 \mu \mathrm{M}$, the relative cell viability of EC9706 and EC1 cells declined to 31.32 and $19.35 \%$, respectively. The cell viability declined to 18.92 and $12.33 \%$ after $48 \mathrm{~h}$ of treatment with 5.0 $\mu \mathrm{M}$ TSA. These data indicated that TSA exhibited its inhibitory effects in ESCC cells in a concentration-dependent manner.

TSA suppresses proliferation of ESCCs by cell cycle arrest. To determine whether the cell growth inhibition effects of TSA were due to cell cycle arrest, the cell cycle phase distribution of ESCC was examined following TSA treatment. There was no notable change in the cell cycle phase distribution of EC9706 at doses of 0.3 or 0.5 . $\mu \mathrm{M}$ after $48 \mathrm{~h}$ of TSA treatment. However, the percentage of untreated EC9706 cells in the G1/G0 phase was $59.86 \%$, which increased to $74.77 \%$ after $48 \mathrm{~h} 1.0 \mu \mathrm{M}$ TSA treatment, while the percentage of S phase cells decreased from 29.09 to $13.03 \%$ (Fig. 2A). In EC1 cells, the percentage of G1/G0 phase cells increased from 53.75 to $78.96 \%$, and S phase cells decreased from 36.63 to $10.24 \%$. These results indicated that a significant G1/G0 arrest was induced in ESCC cells compared with control cells, with a corresponding decrease of cells in the $\mathrm{S}$ phase after $48 \mathrm{~h}$ of treatment $(\mathrm{P}<0.05$; Fig. 2B).

With these results, it was assumed that TSA might selectively affect the expression of G1 cell cycle components. To investigate the mechanism of cell cycle arrest in ESCC cells, we analyzed the protein levels of p21 and p27 via western blot analysis following treatment with control and TSA (0.3 to $1.0 \mu \mathrm{M}$ ). As shown in Fig. 2E, it was observed that TSA induced a significant increase in $\mathrm{p} 21$ and $\mathrm{p} 27$ protein levels at the dose of $1.0 \mu \mathrm{M}$. It has been previously demonstrated that $\mathrm{p} 21$ and p27 participate in negative control of the cell checkpoint by blocking cyclin-dependent kinase (CDK) activity (16). Thus, increases in cellular p21 and p27 correlate with increased inactivation of CDKs. These results suggest that TSA inhibits the proliferation of ESCC lines EC9706 and EC1 by G1 phase cell cycle arrest through an induction of p21 and p27.

TSA suppresses proliferation of ESCCs by cell apoptosis. In addition to modulating cell cycle regulatory proteins to suppress cell growth, it has been previously demonstrated that TSA is capable of inducing cell apoptosis in other tumor cell lines (17). To determine whether TSA induces cell apoptosis 


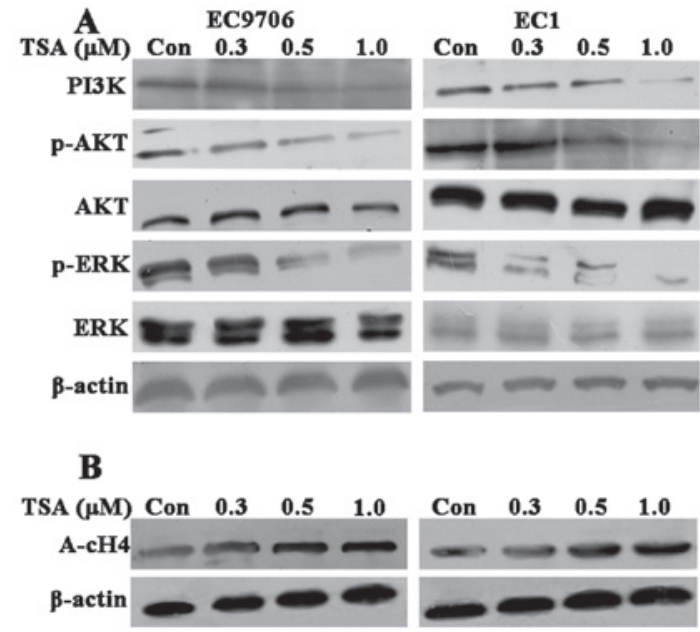

Figure 4. Effects of trichostatin A (TSA) on suppressing the phosphatidylinositol-3-kinase (PI3K)/Akt and mitogen-activated protein kinase (MAPK)/ extracellular signal-regulated kinase (ERK)1/2 signaling pathways. (A) TSA inhibited esophageal squamous cell carcinoma proliferation by suppressing the PI3K/Akt and MAPK/ERK1/2 signaling pathways. EC9706 and EC1 cel lines were treated with TSA at various concentrations $(0.3,0.5$ and $1.0 \mu \mathrm{M})$ for $60 \mathrm{~min}$, then the protein expression levels of PI3K, p-Akt, Akt, p-ERK1/2 and ERK1/2 were detected by western blot analysis. (B) Expression of acetylated histone $\mathrm{H} 4$ was detected after $48 \mathrm{~h}$ of treatment. All experiments were repeated three times.

in ESCC cell lines, EC9706 and EC1 cells were treated with various doses of TSA for $48 \mathrm{~h}$. As shown in Fig. 3A and B, the percentage of early apoptosis was not notably increased at doses of 0.3 and $0.5 \mu \mathrm{M}$ TSA. However, $1.0 \mu \mathrm{M}$ TSA treatment significantly induced early apoptosis compared with that of the control groups $(\mathrm{P}<0.05)$. Furthermore, the percentages of late apoptosis in both EC9706 and EC1 cells increased in a concentration-dependent manner.

To investigate the underlying mechanism of cell apoptosis, the protein levels of apoptosis-related proteins Bcl-2 and Bax were measured before and after TSA treatment. Bcl-2 is an anti-apoptotic protein which inhibits the activity of cysteine proteases known as the ICE family proteases. Significant Bcl-2 downregulation was induced in EC9706 cells and EC1 cells after $48 \mathrm{~h}$ TSA exposure relative to the control groups $(\mathrm{P}<0.05)$. Furthermore, Bcl-2 downregulation occurred earlier in EC1 cells than in EC9706 cells (Fig. 3E). These responses are in accordance with cell apoptosis induced by TSA. Decreased $\mathrm{Bcl}-2$ protein levels may cause an increase in ICE protease activity; thus, ESCC cells may undergo apoptosis. Bax is a pro-apoptotic protein which regulates programmed cell death. The level of Bax significantly increased following 1.0 $\mu \mathrm{M}$ TSA treatment. These results indicate that decreased anti-apoptotic protein Bcl-2 and increased pro-apoptotic protein Bax are underlying apoptotic mechanisms of cell apoptosis.

TSA suppresses the PI3K/Akt and mitogen-activated protein kinase $(M A P K) / E R K 1 / 2$ signaling pathways to inhibit ESCC proliferation. The PI3K/Akt and MAPK signaling pathways including the ERK pathways play a key role in regulating cell growth, proliferation and survival. To investigate the underlying mechanism of TSA on the biological behavior of ESCC cells, we examined the expression of PI3K and the phosphorylation of Akt and ERK1/2 (Fig. 4A). TSA decreased the protein level of PI3K as well as the phosphorylation of Akt and ERK1/2 in a dose-dependent manner without affecting their total protein levels following TSA treatment for $60 \mathrm{~min}$ in the two ESCC cell lines. These results indicated that TSA potently inhibited the proliferation of ESCC by suppressing the PI3K/Akt and ERK1/2 pathways.

To further examine whether the observed effects of ESCC induced by TSA are due to direct modification of histone acetylation we also evaluated the acetylation level of histone $\mathrm{H} 4$ by western blot analysis. The acetylation of histone H4 increased in a concentration-dependent manner following TSA treatment for $48 \mathrm{~h}$ in both EC9706 cells and EC1 cells (Fig. 4B). These results suggested that TSA inhibited their deacetylation. Therefore, the effects of TSA on ESCC proliferation are partly owing to modification of histone acetylation.

\section{Discussion}

ESCC is one of the most aggressive types of malignant cancer with poor prognosis. Epigenetic regulation of gene expression via acetylation of histone and other essential cellular proteins is a potentially useful therapeutic strategy. The HDAC-mediated epigenetic mechanism has a central role in regulating gene expression through chromatin remodeling. HDACs are often overexpressed in numerous types of cancer (18-20). For these reasons, HDAC inhibitors are promising antitumor drugs (21-23). In this study, we evaluated the effects of pan-HDAC inhibitor TSA on cell proliferation, cell cycle regulation and cell apoptosis in ESCC cell lines. Moreover, we also demonstrated that inhibition of HDAC activity with TSA dramatically suppressed the PI3K/Akt and MAPK signaling pathways.

TSA was observed to exert a potent antitumor activity on human colon carcinoma cells and breast adenocarcinoma cells $(24,25)$. TSA was also reported to inhibit the growth of prostate cancer cells through the induction of cell cycle arrest and cell apoptosis (26). In this study, we demonstrated that TSA exhibits its inhibitory effects in ESCC cells in a time- and dose-dependent manner. To further investigate the anti-proliferation mechanisms of TSA toward ESCC, we analyzed the effects of TSA on the cell cycle. G0/G1 arrest was observed in treated EC9706 and EC1 cells. The cell cycle in mammals is controlled by cyclins and CDKs (27-29). CDK inhibitors including p21 and p27 are essential regulators of the cell cycle, inhibiting the activity of cyclin D1/CDK4/6 and cyclin E/CDK2 complexes and blocking cell-cycle transition from $\mathrm{G} 1$ phase to $\mathrm{S}$ phase (30-32). In our study, p21 and p27 were continually upregulated after $48 \mathrm{~h}$ incubation with TSA. These results further demonstrate that TSA exerts its inhibitory effects on cell cycle progression, possibly by inducing $\mathrm{p} 21$ and p27, and this may be the underlying mechanism of the subsequent growth inhibition effect of TSA in ESCC.

In addition to blocking cell-cycle transition, HDAC inhibitors induce apoptosis via the death-receptor apoptosis pathway or the mitochondrial-mediated apoptosis pathway. The mitochondrial pathway disrupts the mitochondrial membrane, causing the release of proteins, including cytochrome $\mathrm{c}$ and other pro-apoptotic molecules, into the cytoplasm $(33,34)$. In our study, TSA enhanced the expression of pro-apoptotic protein Bax and decreased the expression of anti-apoptotic 
protein Bcl-2, thereby activating the mitochondrial-mediated apoptosis pathway.

As is well known, the PI3K/Akt and MAPK signaling pathways are closely related to cell proliferation, differentiation and survival (35-37). It was demonstrated that HDAC inhibitor caused Akt dephosphorylation in three diffuse large B-cell lymphoma cell lines (17). We demonstrated that TSA decreased the expression of PI3K and the phosphorylation of Akt in two ESCC cell lines. We further investigated whether the MAPK signaling pathway is part of the mechanism triggered by TSA to inhibit cell proliferation in ESCC. In our study, TSA reduced the phosphorylation of ERK1/2 in a dose-dependent manner in both EC9706 and EC1 cell lines. These results indicated that suppressing the PI3K/Akt and MAPK signaling pathways in ESCC is partly responsible for the cell growth inhibition induced by TSA.

In conclusion, we demonstrated that the HDAC inhibitor TSA has an anti-proliferative effect in ESCC cell lines via the induction of cell cycle arrest and apoptosis. TSA induces cell cycle arrest in the G1/G0 phases through the induction of p21 and p27. TSA induced apoptosis by decreasing the anti-apoptotic protein $\mathrm{Bcl}-2$ and increasing the pro-apoptotic protein Bax. TSA induces a significant decrease in ESCC cell growth by potently inhibiting the PI3K/Akt and ERK1/2 pathways. Overall, our results suggest that HDAC inhibitors are promising drugs to treat ESCC.

\section{Acknowledgements}

This study was supported by the National Basic Research Program of China (grant no. 2012CB933300), the Natural Science Foundation of the Henan Province of China (grant nos. 12B310023 and 81372269) and the Natural Science Foundation of the Henan province of China (grant nos. 13HASTIT022 and 12B310022).

\section{References}

1. Zhu H, Huo X, Chen L, Wang $\mathrm{H}$ and Yu H: Clinical experience with radio-, chemo- and hyperthermotherapy combined trimodality on locally advanced esophageal cancer. Mol Clin Oncol 1: 1009-1012, 2013.

2. Jemal A, Center MM, DeSantis C and Ward EM: Global patterns of cancer incidence and mortality rates and trends. Cancer Epidemiol Biomarkers Prev 19: 1893-1907, 2010.

3. Minsky BD, Pajak TF, Ginsberg RJ, et al: INT 0123 (Radiation Therapy Oncology Group 94-05) phase III trial of combined-modality therapy for esophageal cancer: high-dose versus standard-dose radiation therapy. J Clin Oncol 20 1167-1174, 2002.

4. Minucci S and Pelicci PG: Histone deacetylase inhibitors and the promise of epigenetic (and more) treatments for cancer. Nat Rev Cancer 6: 38-51, 2006.

5. Reichert N, Choukrallah MA and Matthias P: Multiple roles of class I HDACs in proliferation, differentiation and development. Cell Mol Life Sci 69: 2173-2187, 2012.

6. Ellis L, Atadja PW and Johnstone RW: Epigenetics in cancer: targeting chromatin modifications. Mol Cancer Ther 8: 1409-1420, 2009.

7. Ellis L and Pili R: Histone deacetylase inhibitors: advancing therapeutic strategies in hematological and solid malignancies. Pharmaceuticals (Basel) 3: 2411-2469, 2010.

8. Abbas A and Gupta S: The role of histone deacetylases in prostate cancer. Epigenetics 3: 300-309, 2008.

9. Bonfils C, Walkinshaw DR, Besterman JM, Yang XJ and Li Z: Pharmacological inhibition of histone deacetylases for the treatment of cancer, neurodegenerative disorders and inflammatory diseases. Expert Opin Drug Discov 3: 1041-1065, 2008.
10. Ropero S and Esteller M: The role of histone deacetylases (HDACs) in human cancer. Mol Oncol 1: 19-25, 2007.

11. Cress WD and Seto E: Histone deacetylases, transcriptional control and cancer. J Cell Physiol 184: 1-16, 2000.

12. Meng F, Sun G, Zhong M, Yu Y and Brewer MA: Inhibition of DNA methyltransferases, histone deacetylases and lysine-specific demethylase-1 suppresses the tumorigenicity of the ovarian cancer ascites cell line SKOV3. Int J Oncol 43: 495-502, 2013.

13. Ueki N, Lee S, Sampson NS and Hayman MJ: Selective cancer targeting with prodrugs activated by histone deacetylases and a tumour-associated protease. Nat Commun 4: 2735, 2013.

14. Wilson PM, Labonte MJ, Martin SC, et al: Sustained inhibition of deacetylases is required for the antitumor activity of the histone deactylase inhibitors panobinostat and vorinostat in models of colorectal cancer. Invest New Drugs 31: 845-857, 2013.

15. Chen X, Xiao W, Chen W, Luo L, Ye S and Liu Y: The epigenetic modifier trichostatin A, a histone deacetylase inhibitor, suppresses proliferation and epithelial-mesenchymal transition of lens epithelial cells. Cell Death Dis 4: e884, 2013.

16. Stein C, Riedl S, Rüthnick D, Nötzold RR and Bauer UM: The arginine methyltransferase PRMT6 regulates cell proliferation and senescence through transcriptional repression of tumor suppressor genes. Nucleic Acids Res 40: 9522-9533, 2012.

17. Cai Y, Cui W, Chen W, et al: The effects of a histone deacetylase inhibitor on biological behavior of diffuse large B-cell lymphoma cell lines and insights into the underlying mechanisms. Cancer Cell Int 13: 57, 2013.

18. Spiegel S, Milstien S and Grant S: Endogenous modulators and pharmacological inhibitors of histone deacetylases in cancer therapy. Oncogene 31: 537-551, 2012.

19. Mutze K, Langer R, Becker K, et al: Histone deacetylase (HDAC) 1 and 2 expression and chemotherapy in gastric cancer. Ann Surg Oncol 17: 3336-3343, 2010

20. Jurkin J, Zupkovitz G, Lagger S, et al: Distinct and redundant functions of histone deacetylases HDAC1 and HDAC2 in proliferation and tumorigenesis. Cell Cycle 10: 406-412, 2011.

21. Hoshino I and Matsubara H: Recent advances in histone deacetylase targeted cancer therapy. Surg Today 40: 809-815, 2010.

22. Noureen N, Rashid H and Kalsoom S: Identification of type-specific anticancer histone deacetylase inhibitors: road to success. Cancer Chemother Pharmacol 66: 625-633, 2010.

23. Marson CM: Histone deacetylase inhibitors: design, structure-activity relationships and therapeutic implications for cancer. Anticancer Agents Med Chem 9: 661-692, 2009.

24. Rezaei PF, Fouladdel S, Hassani S, et al: Induction of apoptosis and cell cycle arrest by pericarp polyphenol-rich extract of Baneh in human colon carcinoma HT29 cells. Food Chem Toxicol 50: 1054-1059, 2012.

25. Ward CS, Eriksson P, Izquierdo-Garcia JL, Brandes AH and Ronen SM: HDAC inhibition induces increased choline uptake and elevated phosphocholine levels in MCF7 breast cancer cells. PLoS One 8: e62610, 2013.

26. Watson JA, McKenna DJ, Maxwell P, et al: Hyperacetylation in prostate cancer induces cell cycle aberrations, chromatin reorganization and altered gene expression profiles. J Cell Mol Med 14: $1668-1682,2010$.

27. Gerard C and Goldbeter A: From quiescence to proliferation: Cdk oscillations drive the mammalian cell cycle. Front Physiol 3: 413, 2012

28. Yagi Y, Fushida S, Harada S, et al: Effects of valproic acid on the cell cycle and apoptosis through acetylation of histone and tubulin in a scirrhous gastric cancer cell line. J Exp Clin Cancer Res 29: 149, 2010.

29. Wang C, Fu M, Mani S, Wadler S, Senderowicz AM and Pestell RG: Histone acetylation and the cell-cycle in cancer. Front Biosci 6: D610-D629, 2001.

30. Aguero MF, Facchinetti MM, Sheleg Z and Senderowicz AM: Phenoxodiol, a novel isoflavone, induces G1 arrest by specific loss in cyclin-dependent kinase 2 activity by 553 -independent induction of p21WAF1/CIP1. Cancer Res 65: 3364-3373, 2005.

31. Queiroz AB, Focchi G, Dobo C, Gomes TS, Ribeiro DA and Oshima CT: Expression of p27, p21 (WAF/Cip1) and p16 (INK4a) in normal oral epithelium, oral squamous papilloma and oral squamous cell carcinoma. Anticancer Res 30: 2799-2803, 2010.

32. Tula-Sanchez AA, Havas AP, Alonge PJ, et al: A model of sensitivity and resistance to histone deacetylase inhibitors in diffuse large B cell lymphoma: Role of cyclin-dependent kinase inhibitors. Cancer Biol Ther 14: 949-961, 2013. 
33. You BR and Park WH: Trichostatin A induces apoptotic cell death of HeLa cells in a Bcl-2 and oxidative stress-dependent manner. Int J Oncol 42: 359-366, 2013.

34. Hacker S, Karl S, Mader I, et al: Histone deacetylase inhibitors prime medulloblastoma cells for chemotherapy-induced apoptosis by enhancing p53-dependent Bax activation. Oncogene 30: 2275-2281, 2011 .

35. Zhang J, Roberts TM and Shivdasani RA: Targeting PI3K signaling as a therapeutic approach for colorectal cancer. Gastroenterology 141: 50-61, 2011.
36. Chen CS, Weng SC, Tseng PH, Lin HP and Chen CS: Histone acetylation-independent effect of histone deacetylase inhibitors on Akt through the reshuffling of protein phosphatase 1 complexes. J Biol Chem 280: 38879-38887, 2005.

37. Liu B and Kuang A: Genetic alterations in MAPK and PI3K/Akt signaling pathways and the generation, progression, diagnosis and therapy of thyroid cancer. Sheng Wu Yi Xue Gong Cheng Xue Za Zhi 29: 1221-1225, 2012 (In Chinese). 